\title{
Analysis of mutational pattern in multidrug resistant tuberculosis (MDR TB) in a geographically isolated northeastern region of India
}

\author{
Leimapokpam Shivadutta Singh*, Pranab Behari Mazumder, \\ Gauri Dutt Sharma \\ Department of Biotechnology, Assam University, Silchar \\ Department of Biotechnology, Assam University, Silchar \\ Bilaspur University, Bilaspur
}

\begin{abstract}
Objectives: To assess the mutational pattern of multidrug resistant tuberculosis and evaluate the performance of MTBDRplus and MTBDRsl in rapid detection of multidrug and second line resistance of tuberculosis in a geographically isolated population.

Methods: The assay was performed directly on 375 smear positive sputum specimens from patients referred to a busy routine diagnostic lab in Manipur, northeast India. MTBDRplus andMTBDRsl were performed as per the directions of the manufacturer. Results were compared with BacT/Alert 3D culture and DST system

Results: Of the 375 smear positive specimen, 364 (97.1\%) gave interpretable results by MTBDRplus assay within 1 - 2 days with an additional 2days for EMB resistance of the MDR strains.Sensitivity, specificity, and positive and negative predictive values were high for rifampicin and multidrug resistant strains with a range of $98-100 \%$. Isoniazid has comparatively lower sensitivity of $81 \%$ amongst the drug compared with phenotypic BacT/Alert $3 D$ results. The mutational pattern of this geographically isolated region didn't have much variation as shown by the absence of bands in the rpoB MUT2A/2B and inhA MUT2/3A/3B regions of the gene.

Conclusions: The results of this study supports the use of MTBDRplus for rapid diagnosis of TB from smear positive specimens considering the drastic reduction of time in diagnosis and its accuracy being comparable to phenotypic methods while the MTBDRsl's ability to detect rare mutations makes it a potential method for analysing mutational patterns.
\end{abstract}

Keywords: tuberculosis; MTBDRplus; MTBDRsl; northeast India; Manipur

\section{Introduction:}

India has become the world hub for tuberculosis. According to the latest World Health Organisation (WHO) report titled "Global Tuberculosis Control 2011" 8.8 million cases of TB are registered of which 2.5 million were from India. India alone accounted for an estimated $26 \%$ of all TB cases worldwide and remains a major killer, killing 2 persons every 3 minutes which is nearly 1000/day.

Multidrug resistant tuberculosis (MDR TB) has evolved to become a serious threat to the general public due to limited treatment options (I-Ching et al, 2006; Morgan et al, 2005). In order to have efficient treatment, it becomes imperative that detection be done as early as possible. The emergence of extensively drug resistant tuberculosis (XDR TB) looms large in the backdrop of such scenario with further spread of drug resistance, especially in HIV-infected patients, as was recently reported (Gandhi2006).XDR TB, defined as MDR TB with additional resistance to fluoroquinolone antibiotic and atleast one of the three injectable drug used for MDR TB treatment (Centre $1 \& 2$ ) has been identified in 84 countries with an average proportion of MDR TB cases with XDR TB as $9 \%(6.7-11.2 \%)$ (WHO, 2012).

As such early detection of drug resistances constitutes one of the priorities of TB control programs. Detection of drug resistance has been performed in the past by conventional methods based ondetection of growth of M.tuberculosis in the presence of antibiotics. Conventional indirect methods of detection for drug resistance take $10-12$ weeks for the sample to get drug susceptibility test (DST) report while direct method may be given by $4-6$ week (Canetti et al, 1963; Caviedes et al, 2002; Kent \& Kubica, 1985). However due to the labouriousness of some of this methods and most of all the long period of time necessary to obtain results, new technologies and approaches have been proposed which include both phenotypic and genotypic methods. In many cases, the genotypic methods in particular have been directed towards detection of rifampicin (RIF) resistance, since it is considered a good surrogate marker for MDR TB, especially in settings with high prevalence of MDR TB (I-Ching et al, 2006; Morgan et al, 2005). Genotypic methods have the advantage of a shorter turnaround time, no need for culture of organism, possibility of direct application in clinical samples, lower biohazard risk and the feasibility of automation. However, not all mechanism of drug resistancesare known. Genotypic methods for drug resistance look for the genetic determinants of resistance rather than the 
resistance phenotype and involve two basic steps - nucleic acid amplification by polymerase chain reaction (PCR) to amplify the sections of the M.tuberculosis genome know to be altered in resistant strains and a second step of assessing the amplified products for specific mutations correlating with drug resistance (Garcia de Viedma, 2003; Palomino, 2005). Nearly all RIF resistant strains contain mutation of the rpoB gene while mutations in the $e m b \mathrm{~B}$ gene were associated with ethambutol (EMB) resistance (Cole, 1996; Riccardi et al, 2009). Mutations in $k a t \mathrm{G}$ gene and inh $\mathrm{A}$ gene were relate to the high level and low level isoniazid (INH) resistance respectively (Vilcheze and Jacob, 2007).

The nature and frequency of mutations in the resistant strains vary significantly based on the geographical location (Mokrousov et al, 2002). There is very less information available on specific mutational patterns in India (Sharma et al, 2003), let alone on the underdeveloped and isolated region of northeast India. MDR TB strains have been reported mostly from countries where HIV and TB co-infection is endemic which includes India (Prasad, 2005). The dual challenges to TB and HIV co-infections are particularly pressing in Manipur, a state in the easternmost corner of northeast India which has the highest estimated adult HIV prevalence in India (NACO India, 2012)

The present study is being undertaken with an aim to extend the knowledge of mutational pattern of M.tuberculosis complex by using the MTBDRplus and MTBDRsl(Hain Lifescience) in Manipur, a geographically isolated region of northeast India. The study also evaluates the MTBDR method by comparing with phenotypic culture based method (BacT/Alert 3D) on an attempt to highlight the feasibility of applications in the current situation of $\mathrm{TB}$, its drug resistance and diagnostic trends in northeastern India.

\section{Study settings.}

\section{Material And Methods}

This study was conducted at the Babina Diagnostic Centre in Imphal, Manipur which is one of the most prominent and main referral diagnostic lab of the region. It currently serves a population of 2.7 million.

Testing was performed on residual portions of routine clinical specimens submitted for culture and DST. Informed consent was not taken for the study as results were unlinked from patient identifiers and no patient information was collected. Only one sample per patient was collected.

Sputum specimens

Three hundred and seventy five sputum samples were collected between May - December 2011. Every handling of the clinical specimens was conducted inside a Class II safety cabinet in aBSL2 laboratory in accordance with CDC guidelines. Sputum decontaminations were carried out with the conventional N-acetyl-Lcysteine- $\mathrm{NaOH}$. After centrifugation, the pellet was suspended in $1 \mathrm{ml}$ of $85 \% \mathrm{NaCl}$. All specimens were processed for acid fast microscopy using Ziel-Neesen technique (Canetti et al, 1963; Kent \& Kubica, 1985). Sputum samples showing more than 10 acid fast bacilli (AFB) per microscopic field in the smear were selected for the study.

\section{BacT/Alert 3D culture and DST}

Drug susceptibility testing for isoniazid, rifampicin and ethambutol was performed by BacT/Alert 3D system. A $0.5 \mathrm{ml}$ portion of the sediment was inoculated into the vials of BacT/Alert 3D system containing modified Middlebrook 7H9 media with respective antibiotic. The final drug concentration in the test bottles was $1 \mu \mathrm{g} / \mathrm{ml}$ for INH and RIF and $21 \mu \mathrm{g} / \mathrm{ml}$ for EMB. All mycobacterial cultures were incubated at $37^{\circ} \mathrm{C}$ and were continuously monitored.M.tuberculosis isolate was determined to be resistant to the antibiotic when the drug containing bottle had a time to detection (TTD) that was less than or equal to the TTD of $1 \%$ control.

Genotype MTBDRplus and MTBDRsl DST

The MTBDRplus and MTBDRsl DST were performed according to the manufacturer's instruction and are divided into three steps: DNA extraction, a multiples amplification using biotinylated primers and reverse hybridisation. A $500 \mu 1$ portion of the decontaminated sediment was used for DNA extraction using modified CTAB-NaCl method. The isolated DNA samples from the process mentioned above were used for multiplex amplification with biotinylated primers. The biotin labelled PCR product was chemically denatured and hybridised to the strip with specific oligonucleotide probes. After hybridisation and washing, strips were removed, allowed to dry and fixed on the nitrocellulose paper.

All samples that tested positive for MDR in the MTBDRplus strip was further subjected to MTBDRsl test. Phenotypic DST was conducted for EMB only in the MDR strains which has been subjected MTBDRsl. All tests were performed independent of culture and DST.

\section{Interpretation of results}

Each strip in MTBDRplus consist of 27 reaction zones (bands) including six controls (conjugate , amplification, M.tuberculosis complex, rpoB, kat $\mathrm{G}$ and inhA controls), eight rpoB wild type (WT) and four mutant (MUT) probes, one katG wild type and two mutant and two inhA wild type and four mutant probes 
(Figure 1a). Each strip in the MTBDRsl consists of 22 reaction zones including six controls (conjugate, amplification, M.tuberculosis complex, gyrA, rrs and embB controls). Three gyrA wild type and six mutant probes, two $r r s$ wild type and two mutant probes and one $e m b \mathrm{~B}$ wild type and two mutant probes (Figure 1b). Results were interpreted according to the manufacturer's instructions.

Statistical methods were performed using SPSS 17.0. Results were considered significant at $\mathrm{P}$ value less than 0.5

\section{Results}

Genotype MTBDRplus testing from smear positive sputum

Genotype MTBDRplus test results in comparison with BacT/Alert 3D DST of all smear positive specimens tested $(n=375)$ are summarised in Table 1 . Fifteen specimens $(4 \%)$ were culture negative and therefore no phenotypic DST results were available. Of these 15 culture negative specimens, $14(93.33 \%)$ gave interpretable results by the MTBDRplus method. Of the specimens with conventional DST results, $60(16.67 \%)$ were NDR, 20(5.6\%) were RIF monoresistant, 14(4.44\%) were INH monoresistant and 366(73.33\%) were RIF and INH susceptible. In comparison with BacT/Alert culture and DST results (96\%), a higher proportion of MTBDRplus results (97.1\%) were interpretable. This genotypic method detected 58 (97\%) MDR, 1 RIF monoresistant with one uninterpretable result out of the 60 MDR strains detected by phenotypic DST. The results for RIF monoresistant was concordant with the phenotypic DST except for one strain which was detected as RIF and INH susceptible out of the 20 RIF monoresistant by phenotypic DST. MTBDRplus detected 269 susceptible strains including 13 culture negative strains while BacT/Alert system could detect 264 susceptible strains including 9 uninterpretable strains of MTBDRplus. The sensitivity, specificity, accuracy, positive predictive values (PPV) and negative predictive values (NPV) are given in Table 2.

BacT/Alert culture and DST for smear positive specimens had a total turnaround time of $8-12$ days. For MTBDRplus testing, the test took $1-2$ days for smear positive results while MTBDRsl testing of the MDR strains took another 2 days.

Table 3 shows the distribution of different banding patterns in drug resistant isolates, including MDR, INH monoresistant and RIF monoresistant strains while Table 4 shows the banding pattern of MDR isolates which has been subjected to MTBDRsl test. Typical banding patterns obtained on MTBDRplus and MTBDRsl strips are shown in Figure 1.

The RIF resistant isolates displayed different mutations. The most common mutation was in the S531L region (MUT3) with $45.6 \%$ of all RIF resistant strains (55\% of MDR and 19\% of RIF monoresistant strains) having the mutation in the $k a t \mathrm{G}$ codon with amino acid change of Ser-315-Thr1. This difference in prevalence of the S531L mutation between MDR and RIF monoresistant strain was significant $(P=0.012)$. Two MDR strain had a D516V mutation (3.4\% overall). Of the isolates with mutation in the wild type probes, mutation in the WT8 was most common, as detected by the lack of binding to the WT8 probe in the absence of S531L mutation except for one strain which had both the WT8 wildtype and the S531L mutation. No mutation was detected in the MUT2A andMUT2B band in both the RIF monoresistant and MDR strains. There was no significant difference in the presence of other bands between MDR and RIF monoresistant strains. Of all the INH resistant strains, $73 \%$ (71\% of MDR and $81 \%$ of INH monoresistant strains) had a mutation in the katG S315T1 region while 4\% had a mutation in the S315T2 region indicating high level resistance while 19\%(19\% Of MDR and 19\% of INH monoresistant strains) had mutation in the C15T region of inhA gene indicating low level resistance. This difference in prevalence of mutations in MDR strains compared with INH monoresistant strains was significant for $k a t \mathrm{G}(\mathrm{P}=0.0073)$ but not for inh $\mathrm{A}(\mathrm{P}=0.1497)$. Five strains had mutations in both the $k a t \mathrm{G}$ andinhAgenes. Seventy six percent (28/37) of MDR strains had a mutation in the kat $\mathrm{G}$ gene and were detected as INH resistant y a mutation in the $k a t$ G gene. Only three of $16(18.8 \%)$ INH monoresistant strains were detected by the presence of a mutation in inhA only

Genotype MTBDRsl testing from MDR positive samples.

All samples tested for XDR by MTBDRsl were fortunately negative which indicates that XDR is not yet detected from this region. This non-detection of XDR was all due to no mutation being detected in the rrs gene probe while mutation was detected in all the mutation region of gyrA gene with the highest rate in the D94G region (17\%). One MDR positive strain had a gyrA MUT3D mutation. This is a rare mutation which has been detected only in silico and hence thought to be undetectable in vitro. But this detection albeit in one strip proves that it is detectable and that the MTBDRsl is efficient in detecting even rare mutations. Of the $32 \mathrm{EMB}$ resistant strains according to the molecular method, 5 strains had mutations in the embB gene region of codon 306 with amino acid change of Met-306-Ile and 27 strain had mutation in the embB gene with amino acid change of Met-306-Val. One strain had mutation that was detected only at the wild type probes (embB WT) but not at the mutant probes.

Performance of Genotype MTBDRplus and Genotype MTBDRsl assays 
Performance parameters for detection of INH/RIF monoresistant, MDR and EMB resistance were calculated from specimens for which genotypic and phenotypic DST results were available (Table 2). The performance of the genotypic MTBDRplus test directly from smear positive sputum correlated highly with the phenotypic BacT/Alert automated DST. Compared with the phenotypic DST, the genotypic MTBDRplus had a sensitivity of $81.3 \%$, specificity of $99.2 \%$ and accuracy of $98.2 \%$ for detection of INH, a sensitivity of $95 \%$, specificity of $100 \%$ and accuracy of $99.6 \%$ for RIF resistance and a sensitivity of $98.3 \%$, specificity of $100 \%$ and accuracy of 99.7\% for MDR. The genotypic MTBDRsl assay for ethambutol had a sensitivity of $86 \%$, specificity of $91 \%$ and accuracy of $88 \%$ for detection of EMB resistance.

\section{Discussion}

The efficiency of Genotype MTBDRhave been reported by many authors (Hilleman et al, 2006, 2007; Barnard et al,2007, 2008) having good concordance with phenotypic DST results but presence of false negative due to unique mutation are also reported (Hilleman et al.2007;Brossier et al,2006;Tessema et al 2012)). This may be due to specific location independently developing endemic mutations. The present study evaluates the INH,RIF,MDR and EMB mutations using genotypic MTBDRplus and MTBDRsl test compared with BacT/Alert 3D DST of a geographically isolated region of northeast India. The results of the study showed that the mutational pattern of the resistant genes didn't vary much with most mutations confined to one or two main regions. It is seen that in this region of India, the most frequent mutation causing RIF resistance is the Ser-531Leu mutation(45.6\%) and in INH resistance, the Ser-315-Thr1 is the most common(19\%). This is more or less in agreement with earlier reported studies (Miotto et al, 2006; Mokrousov 2002). In case of EMB resistance, Met-306-Val mutation (45\%) is observed to be the major cause. Previous studies have shown that 40-95\% of isoniazid resistance are defined as the high level drug resistance due to $\mathrm{kat} \mathrm{G}$ gene mutations. In this present study, 77\% of INH resistance are attributed to kat $\mathrm{G}$ mutations of which 95\% are S315T1 and 5\% are S315T2 mutations. A high prevalence of $k a t \mathrm{G}$ mutations has been reported to account for a high proportion of INH resistance in high TB prevalent countries presumably due to ongoing transmission of these strains in high burden setttings (Mokrousov et al 2002). India, being one of the top high TB burden countries, such high prevalence was expected.

The performance characteristics of specificity, sensitivity, accuracy, PPV and NPV as given in Table 2 suggest that the MTBDRplus assay is equivalent to phenotypic culture based DST performed in quality assured reference laboratories but detection of ethambutol resistance by MTBDRsl, though lower than INH or RIF detection by MTBDRplus, is comparatively higher than other reported studies (Brossier et al 2010; Tesema et al 2012). The lower value of sensitivity for detection of INH resistance $(81.25 \%)$ by genotypic method might be due to resistance inferred by mutations in other genomic region which has not been incorporated in this assay, such as $n d h$ andkasA gene (Lee et al, 1999, 2001; Rindi et al, 2005). Three out of 16 INH monoresistant strains were detected by mutations in inhA gene only. These mutations would not have been detected by the previous MTBDR which didn't incorporate the inhA probe. As such, mutations in other genomic regions might be inducing resistance to INH which would not be detected by genotypic method if their corresponding probes were not included, leading to false positives apart from the false negative results due to presence of inhibitors (Palomino, 2006).

Identification and drug resistance assay of M.tuberculosis normally requires several weeks as they are very slow in their growth and even with automated culture system, it takes an average of 14 days. Another 14 days for additional tests are required for DST. Moreover, the culture based methods involve increased consumption of exclusive culture media and hence place more economic burden on patients especially in a low resource and high burden region like Manipur. On the basis of FIND-negotiated prices, the cost of molecular assay is less than $50 \%$ of that for conventional liquid culture and DST for INH and RIF (Barnard et al,2008) Therefore there is urgency for an efficient method of TB testing as a complement to conventional culture and smear microscopy.

In conclusion, the results of this study supports the use of MTBDRplus for rapid diagnosis of TB considering the drastic reduction of time in diagnosis and its accuracy being comparable to conventional methods. It might also be more cost effective in the long run. This molecular genotyping will also be useful in studying epidemiological and mutational analysis of M.tuberculosis of specific regions.

\section{References}

[1]. Barnard M, Albert H, O'Brien R, Coetzee G, Bosman M. Assessment of the MTBDRPlus assay for the rapid detection of multidrug-resistant tuberculosis from smear-positive sputa. Int J Tuberc Lung Dis 2007; 11:S229.

[2]. Barnard M, Albert H, Coetzee G,O;Brien R, Bosman M. Rapid molecular screening for multidrug-resistant tuberculosis in a highvolume public health laboratory in South Africa. Am. J. Respir. Crit. Care. Med. 2008; 177: 787-792

[3]. Brossier F, Veziris N, Tuffot-Pernot C, Jarlier V, Sougakoff W. Performance of the Genotype MTBDR line probe assay for detection of resistance to rifampicin and isoniazid in strains of Mycobacterium tuberculosis with low and high level resistance. J. Clin. Microbiol. 2006; 44: 3659 - 3664 
[4]. Brossier F, Veziris N, Aubry A, Jarlier V, Sougakoff W. Detection by Genotype MTBDRsl test of complex mechanism of resistance to second-line drugs and ethambutol in multidrug-resistant Mycobacterium tuberculosis complex isolates. J. Clin. Microbiol. 2010; 48:1683 - 1689

[5]. Canetti G, Froman S, Grosset J, Haudway P, Langerova M, Mahler H T, Meissner G, Mitchison D A and Sula L. Mycobacteris: Laboratory methods for testing drug sensitivity and resistance. Bull. World Health Organ. 1963; 29: 565 - 578

[6]. Caviedes L, Deelgado J and Gilman R H. Tetrezoliummicroplate assay as rapid and inexpensive clorimetric method for determination of antibiotic susceptibility of Mycobacterium tuberculosis. J. Clin. Microbiol. 2002; 20(40:5):1873 - 1874

[7]. Centers for Disease Control and Prevention. Emergence of Mycobacteriumtuberculosis with extensive resistance to second-line drugs worldwide, 2000-2004. MMWR Morb Mortal Wkly Rep 2006;55:301-305.

[8]. Centers for Disease Control and Prevention. Notice to readers: revised definition of extensively drug-resistant tuberculosis. MMWR Morb Mortal Wkly Rep 2006;55:1176.

[9]. Cole S T. Rifampicin resistance in Mycobacteria. Res. Microbial. 1996; 147:48 - 52

[10]. Gandhi NR, Moll A, Sturm AW, et al. Extensively drug-resistant tuberculosis as a cause of death in patients co-infected with tuberculosis and HIV in a rural area of South Africa. Lancet 2006; 368: 1575-80.

[11]. Garcia de Viedma, D. Rapid detection of resistance in Mycobacterium tuberculosis: a review discussing molecular approaches. Clin. Microbiol. Infect. 2003; 9, 349-359.

[12]. GenoType MTBDRplus, Nehren, Germany: Hain Lifescience, GmbH. Available from: http://www.hain-lifescience.com

[13]. GenoType MTBDRsl, Nehren, Germany: Hain Lifescience, GmbH. Available from: http://www.hain-lifescience.com

[14]. Hilleman D, Rusch-Gerdes S, Richter E. Application of the Genotype MTBDR assay directly on sputum samples. Int. J.Tuberc. Lung. Dis. 2006; 10:1057-1059.

[15]. Hilleman D, Ru“ sch-Gerdes S, Richter E. Evaluation of the Genotype MTBDRplus Assay for rifampicin and isoniazid susceptibility testing of Mycobacterium tuberculosis strains and in clinical specimens. J Clin.Microbiol 2007;45:2635-2640.

[16]. I-Ching s, Drobniewski F, More P, Kemp M and Brown T. Mycobacterium tuberculosis and rifampicin. Dis. 2006; 12(5):752 - 759

[17]. Kent, P.T., Kubica, G.P. Public Health Mycobacteriology. A Guide for the Level III Laboratory. US Department of Health and Human Services, Centers for Disease Control and Prevention, Atlanta. 1985.

[18]. Lee A S G, Lim I H K, Tang L L H, Telenti A, Wong S Y. Contribution of kasA analysis to detection of isoniazid-resistant Mycobacterium tuberculosis in Singapore. Antimicrob Agents Chemother 1999; 43: 2087-2089.

[19]. Lee A S G, Teo A S M, Wong S Y. Novel mutations in $n d h$ in isoniazid-resistant Mycobacterium tuberculosis isolates. Antimicrob Agents Chemother 2001; 45: 2157-2159.

[20]. Miotto, P., F. Piana, V. Penati, F. Canducci, G. B. Migliori, and D. M. Cirillo. Use of GenoType MTBDR assay for molecular detection of rifampin and isoniazid resistance in Mycobacterium tuberculosis clinical strains isolated in Italy. J. Clin. Microbiol. 2006; 44:2485-2491.

[21]. Mokrousov I, Narvskaya O, Otten T, Limenschenko E, Steklova L, Vyshnevskiy B. High prevalence of katG Ser315Thr substitution among isoniazid-resistant Mycobacterium tuberculosis clinical isolates from Northwestern Russia, 1996-2001. Antimicrob Agents Chemother 2002;46:1417-1424.

[22]. Morgan M, Kalantri S, Flores L, Pai M. A commercial line probe assay for the detection of rifampicin resistance in Mycobacterium tuberculosis: a systematic review and meta-analysis. BMC Infectious diseases. 2005; 5:62 - 71

[23]. National AIDS Control Organisation (NACO), Dept. of AIDS control, Ministry of Health and Family Welfare. Annual Report 2011-2012

[24]. Palomino, J.C. Nonconventional and new methods in the diagnosis of tuberculosis: feasibility and applicability in the field. Eur. Respir. J. 2005; 26:1-12

[25]. Palomino, J.C. Newer diagnostics for tuberculosis and multi-drug resistant tuberculosis. Curr. Opin. Pulm. Med. 2006; 12:172-178.

[26]. Prasad R. MDR-TB: current status. Indian. J. Tub.2005; 52:121 - 131

[27]. Riccardi G, Pasca MR,„Buroni S. Mycobacterium tuberculosis: drug resistance and future perspectives. Future Microbiol. 2009; 4:597- 614

[28]. Rindi L, L Bianchi, E Tortoli, N Lari, D Bonanni and C Garzelli. Mutations responsible for Mycobacterium tuberculosis isoniazid resistance in Italy. Int. J. Tuberc. Lung Dis.2005; 9(1): 94 - 97

[29]. Sharma M, Sethi S, Mishra B, Sengupta C and Sharma S K. Rapid detection of mutations in rpoB gene of rifampicin resistant Mycobacterium tuberculosis strains by line probe assay. Indian J. Med. Res. 2003; 117: $76-80$

[30]. Tessema B, Beer J, Emmrich F, Sack U and Rodloff AC. Analysis of gene mutations associated with isoniazid, rifampicin and ethambutol resistance among Mycobacterium tuberculosis isolates from Ethipia. BMC Infectious Diseases. 2012; 12:37

[31]. Vilcheze C and Jacobs WR Jr. The mechanism of isoniazid killing: clarity through the scope of genetics. Annu. Rev. Microbiol. $2007 ; 61: 35-50$

[32]. World health Organisation. Global tuberculosis control report. Geneva, Switzerland: WHO, 2011.

[33]. World health Organisation. Global tuberculosis control report. Geneva, Switzerland: WHO, 2012.

Table 1.Results of MDR by Genotype MTBDRplus in comparison with BactT/Alert culture and DST. Genotype MTBDRplus

\begin{tabular}{lccccc} 
& \multicolumn{4}{c}{ Bact T/Alert 3D and DST } \\
\cline { 2 - 5 } & MDR & RIF monoresistant & INH monoresistant & $\begin{array}{c}\text { RIF \& INH } \\
\text { susceptible }\end{array}$ & $\begin{array}{c}\text { Culture } \\
\text { negative }\end{array}$ \\
\hline MDR & 58 & 0 & 0 & 0 & 0 \\
RIF monoresistant & 1 & 19 & 0 & 1 & 0 \\
INH monoresistant & 0 & 0 & 13 & 3 & 2 \\
RIF \& INH susceptible & 0 & 1 & 0 & 9 \\
Uninterpretable & 1 & 0 & 13 & 1 \\
\hline
\end{tabular}

Definition of abbreviation: DST = Drug Susceptibility Test; RIF = Rifampicin; INH = Isoniazid; MDR = Multidrug resistant 
Analysis of mutational pattern in multidrug resistant tuberculosis (MDR TB) in a geographically

Table 2. Performance of MTBDRplus in detecting MDR from smear positive sputum specimens

\begin{tabular}{lcccc}
\hline Parameters & RIF & INH & MDR & EMB \\
\hline Sensitivity & $95.0(93.0-97.0)$ & $81.25(78.0-85.0)$ & $98.3(97.2-99.5)$ & $85.71(82.6-88.8)$ \\
Specificity & $100(99.0-100)$ & $99.21(98.4-99.0)$ & $100(99.0-100)$ & $91.3(88.8-93.8)$ \\
Accuracy & $99.63(99.1-100)$ & $98.15(97.0-99.3)$ & $99.63(99.1-100)$ & $87.93(85.0-90.8)$ \\
PPV & $100(99.0-100)$ & $86.677(84.0-90.0)$ & $100(99.0-100)$ & $93.75(91.6-995.9)$ \\
NPV & $99.6(99.1-100)$ & $98.82(97.0-99.3)$ & $99.6(99.0-100)$ & $80.77(85.0-90.8)$ \\
\hline
\end{tabular}

Definition of abbreviations: NPV = negative predictive value; PPV = positivepredictive value.

Values are percentages with $95 \%$ confidence interval in parentheses.

Table 3. Pattern of gene mutations in resistant $M$. tuberculosis strains using Genotype MTBDRplus assay

\begin{tabular}{|c|c|c|c|c|c|}
\hline Gene & Band & $\begin{array}{c}\text { Gene region of } \\
\text { mutation }\end{array}$ & $\begin{array}{c}\text { MDR } \\
(n=58)\end{array}$ & $\begin{array}{c}\text { INH } \\
\text { Monoresistant } \\
(n=16)\end{array}$ & $\begin{array}{c}\text { RIF } \\
\text { Monoresistant } \\
(\mathbf{n}=\mathbf{2 1})\end{array}$ \\
\hline \multirow[t]{12}{*}{ rpoB } & WT1 & $505-509$ & $54(93)$ & $16(100)$ & $18(86)$ \\
\hline & WT2 & $510-513$ & $54(93)$ & $16(100)$ & $21(100)$ \\
\hline & WT3 & $513-517$ & $49(84)$ & $16(100)$ & 19(91) \\
\hline & WT4 & $516-519$ & $54(93)$ & $16(100)$ & $19(91)$ \\
\hline & WT5 & $518-522$ & $56(97)$ & $16(100)$ & $19(91)$ \\
\hline & WT6 & $521-525$ & $49(84)$ & $16(100)$ & $13(62)$ \\
\hline & WT7 & $526-529$ & $49(84)$ & $16(100)$ & $12(57)$ \\
\hline & WT8 & $530-533$ & $13(22)$ & $16(100)$ & $0(0)$ \\
\hline & MUT1 & D516V & $2(3)$ & $0(0)$ & $0(0)$ \\
\hline & MUT2A & H526Y & $0(0)$ & $0(0)$ & $0(0)$ \\
\hline & MUT2B & H526D & $0(0)$ & $0(0)$ & $0(0)$ \\
\hline & MUT3 & S531L & $32(55)$ & $0(0)$ & $4(19)$ \\
\hline \multirow[t]{3}{*}{ katG } & WT & 315 & $13(22)$ & $2(13)$ & $21(100)$ \\
\hline & MUT1 & S315T1 & $41(71)$ & $13(81)$ & $0(0)$ \\
\hline & MUT2 & $\mathrm{S} 315 \mathrm{~T} 2$ & $2(3)$ & $1(6)$ & $0(0)$ \\
\hline \multirow[t]{6}{*}{$\operatorname{inh} A$} & WT1 & $-15 /-16$ & $47(81)$ & $13(81)$ & $21(100)$ \\
\hline & WT2 & -8 & $55(95)$ & $15(94)$ & $21(100)$ \\
\hline & MUT1 & C15T & 11(19) & $3(19)$ & $0(0)$ \\
\hline & MUT2 & A16G & $0(0)$ & $0(0)$ & $0(0)$ \\
\hline & MUT3A & $\mathrm{T} 8 \mathrm{C}$ & $0(0)$ & $0(0)$ & $0(0)$ \\
\hline & MUT3B & T8A & $0(0)$ & $0(0)$ & $0(0)$ \\
\hline
\end{tabular}

Definition of abbreviations: RIF = rifampicin; INH = isoniazid; $\mathrm{MDR}=$ multidrug resistant;

Values are numbers, with percentages in parentheses.

Table 4. Pattern of gene mutations in MDR M. tuberculosis strains using Genotype MTBDRsl assay

\begin{tabular}{cccc}
\hline Gene & Band & Gene region of mutation & $\begin{array}{c}\text { Pattern of mutation } \\
(\mathbf{n}=\mathbf{5 8})\end{array}$ \\
\hline gyrA & WT1 & $85-90$ & $58(100)$ \\
& WT2 & $89-93$ & $51(88)$ \\
& WT3 & $92-97$ & $44(75)$ \\
& MUT1 & A90V & $8(14)$ \\
& MUT2 & S91P & $3(5)$ \\
& MUT3A & D94A & $5(9)$ \\
& MUT3B & D94N/Y & $5(9)$ \\
& MUT3C & D94G & $10(17)$ \\
rss & MUT3D & D94H & $1(2)$ \\
& WT1 & $1401-1402$ & $24(100)$ \\
& WT2 & 1484 & $24(100)$ \\
embB & MUT1 & A1401G/C1402T & $0(0)$ \\
& MUT2 & G1484T & $0(0)$ \\
& WT1 & 306 & $25(43)$ \\
& MUT1A & M306I & $5(9)$ \\
& MUT1B & M306V & $27(47)$
\end{tabular}

Values are numbers, with percentages in parentheses. 


\section{Figure 1.}

1a. Examples of GenoTypeMTDBRplus strips (Hain Lifescience, Nehren, Germany). (Lane 1) Multidrugresistant tuberculosis (MDR TB), rpoB S531L mutation and inhA C15T mutations. (Lane 2) MDR TB, rpoB S531L mutation and katG S315T1 mutation. (Lane 3) M.tuberculosis, INH monoresistant (katG S315T1 mutation). (Lane 4) Mycobacterium tuberculosis, RIF monoresistant (rpoB S531L mutation in 530-533 region). (Lane 5) M. tuberculosissusceptible to isoniazid (INH) and rifampin (RIF). (Lane 6) Negative control.

1b. Examples of Genotype MTBDRsl (Lane 1) MDR Tb with gyrA WT3/ D94G and embB M306V mutation (Lane 2) gyrA WT2/S91P and embB M3061 mutation (Lane 3) gyrA WT3/D94NY/D94G and embB M306V mutation (Lane 4) MDR TB susceptible to Flouroquinone, Cyclic peptides and Ethambutol (Lane 5) Negative control.
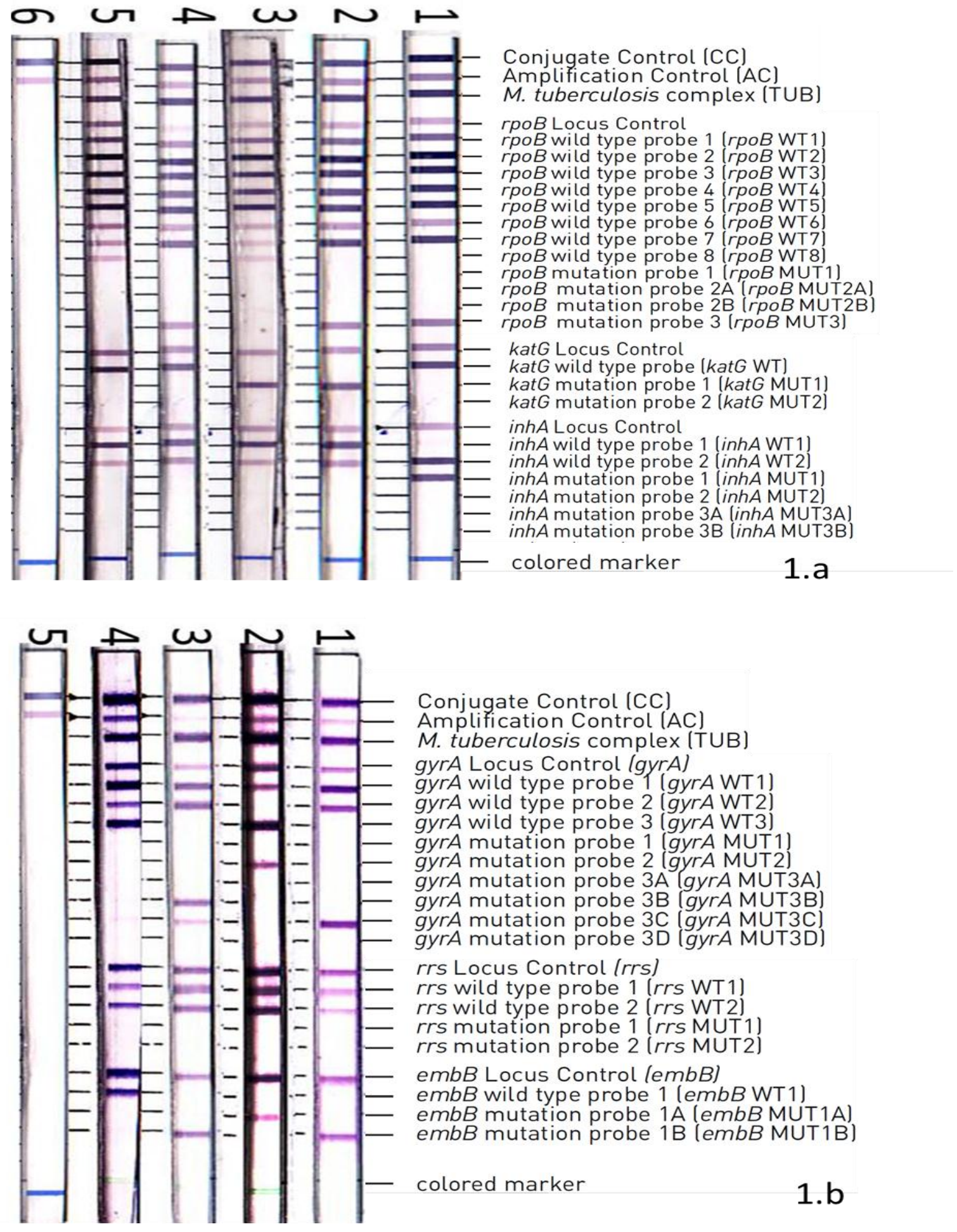